\title{
A novel hybrid approach to detect brain tumor in MRI images
}

\author{
Tejas $\mathbf{P}^{\mathrm{a}}$, S.K. Padma \\ a,b Department of Information Science and Engineering, Sri Jayachamarajendra College of Engineering, Mysuru, 57006 (K. \\ A.), INDIA \\ atejaskp114@gmail.com, ${ }^{\mathrm{b}}$ skp@sjce.ac.in
}

Article History: Received: 10 November 2020; Revised 12 January 2021 Accepted: 27 January 2021; Published online: 5 April 2021

\begin{abstract}
Image segmentation is the fundamental step in medical image analysis. Segmentation is a procedure to separate similar portions of images showing resemblance in different features such as color, intensity, or texture. Grayscale images are mostly used for the segmentation of medical images. Tumors are commonly stated as the abnormal growth of tissues and the brain tumor is a diseased part in the body tissues that is an abnormal mass in which the growth rate of cells is irrepressible. The mortality rate of people has raised over the past years due to brain tumors, hence this area has gained the attention of researchers. Automatic detection of brain tumors is a challenging task because it involves pathology, functional physics of MRI along with intensity and shapes analysis of MR image. After all, tumor shape, size, location, and intensity vary for each infected case. In this work, a novel hybrid approach is implemented by combing watershed segmentation, level set segmentation and $\mathrm{K}$ means clustering. First, the image is preprocessed by removing the skull. Watershed segmentation is applied to this preprocessed image. Level set segmentation is applied to the previous step. Finally, $\mathrm{k}$ means clustering is applied as the final step to detect tumor parts accurately. This Hybrid approach is compared with other four techniques such as Threshold segmentation, K means clustering, Watershed segmentation, and Level set-based segmentation methods. Statistical and Visual analysis is performed. It is found that the hybrid approach has better specificity, accuracy, and precision among all four techniques. Further, it is able to detect tumors more accurately. This research could help clinicians in surgical planning, treatment planning and accurately segmenting the tumor part with the most accurate method.
\end{abstract}

Keywords: Brain tumor detection, Image segmentation, Threshold segmentation, $\mathrm{K}$ means clustering, Watershed segmentation, Level set segmentation, Hybrid segmentation, novel hybrid approach

\section{Introduction}

Image segmentation is the fundamental step in medical image analysis. Segmentation is a procedure to separate similar portions of images showing resemblance in different features such as color, intensity, or texture [1]. Grayscale images are mostly used for the segmentation of medical images.

Tumors are commonly stated as the abnormal growth of tissues [2] and the brain tumor is a diseased part in the body tissues that is an abnormal mass in which the growth rate of cells is irrepressible [3]. The mortality rate of people has raised over the past years due to brain tumors, hence this area has gained the attention of researchers. Commonly a tumor could be benign or malignant. Benign tumors are those tumors that remain within the boundaries of the brain, whereas the malignant tumors could extend beyond the brain and affect other parts of the body. These kinds of tumors may not be treated because of their aggressive nature. Nowadays imaging is playing a vital role in the diagnosis of a brain tumor in the early stages before they become intractable, thus saving many lives. Different techniques have been developed to detect tumors, like CT, MRI, EEG (electroencephalography), etc. The MR imaging method is the best due to its higher resolution and enhanced quality [4]. Automatic detection of brain tumors is a challenging task because it involves pathology, functional physics of MRI along with intensity and shapes analysis of MR image, because tumor shape, size, location, and intensity vary for each infected case [5].

Image segmentation algorithms are based on gray-level values of the pixels, sudden changes in the gray-level and similarity between pixels regions are the basis for segmentation of an image [6]. Various methods have been proposed for the segmentation of brain tumor from MR images, Baidya Nath Saha et.al [7] presented a bounding box method using symmetry to segment out tumors from brain MR images, Knowledge-based techniques presented by Matthew C. Clark et.al [8] describe and compare results based on supervised and unsupervised clustering. C.L. Biji et.al [9] proposed a fuzzy thresholding technique for brain tumor segmentation. Jianping Fan et.al [10] proposed a seeded region growing method in which seed selection and pixel labeling problems are addressed. Yu-len Huang and Dar-ren Chen [11] proposed segmentation based on a Watershed method for identifying breast tumors. Nelly Gordillo et.al. [12], presented a review of the most relevant brain tumor 
segmentation methods. Accordingly, semi-automated and fully automated segmentation methods have gained importance due to accuracy in identification, but it's a fact that the end systems are used by the physicians therefore there is a surprising lack of compatibility between large computer vision-based frameworks and the lowlevel methods employed for segmentation. The other reason is that these approaches are still not capable to gain acceptance among pathologists for everyday clinical tasks due to not having any standardized procedures. Therefore, these approaches need to be compared with real-world medical issues to address problems of segmentation with the best approaches.

A threshold-based method is a simple and effective segmentation method by comparing their intensities with one or more intensity thresholds. The basic principle of the watershed segmentation method can be explained by a metaphor-based on the behaviour of water in a landscape. When it rains, drops of water falling in different regions will follow the landscape downhill. The water will end up at the bottom of the valleys. For each valley, there will be a region from which all water drains it. At points where water comes from different basins meets, dams will be built. When the water level reaches the highest peak in the landscape, the process is stopped. As a result, the landscape is partitioned into regions separated by dams, called watershed lines or watersheds. Some researchers used multi-scale watershed transformation to segment brain tumors [13, 14].

$\mathrm{K}$-means is a widely used clustering algorithm to partition data into $\mathrm{k}$ clusters. Clustering is the process of grouping data points with similar feature vectors into a single cluster and grouping data points with dissimilar feature vectors into different clusters [15]. Level-sets methods rely on partial differential equations (PDEs) to model deforming isosurfaces. Level set methods rely on two central embeddings; first the embedding of the interface as the zero-level set of a higher dimensional function, and second, the embedding (or extension) of the interface's velocity to this higher dimensional level set function [16].

A comparison between Threshold-based, $\mathrm{K}$ means clustering-based, Watershed based and Level set-based segmentation methods were performed to detect the tumor region of the brain. Statistical and Visual analysis is performed to figure out the best method. Threshold-based segmentation was one of the simplest methods to implement. However, it was not able to distinguish the tumor part properly since most of the time Thresholdbased segmentation is used as a pre-processing step. K-means clustering was not able to detect tumors that are small and which are light in appearance. Watershed segmentation had the highest accuracy in detecting tumor parts compared to all four techniques. It was able to detect almost the exact tumor shape but not better than the level set segmentation. Level set segmentation had the second-highest accuracy among all and was able to detect the exact tumor shape but it took the longest time to display results (3-10 seconds of delay). The Level set also misclassified the tumor more i.e. detecting non-existing tumor. Finally, based on the statistical and visual analysis, it is being concluded that the Watershed segmentation technique was the best among all the segmentation techniques [17].

In this work, a novel hybrid approach is implemented by combing watershed segmentation, level set segmentation and $\mathrm{k}$ means clustering. First, the image is preprocessed by removing the skull. Watershed segmentation is applied to this preprocessed image. Level set segmentation is applied to the previous step. Finally, $\mathrm{k}$ means clustering is applied as the final step to detect tumor parts accurately. This Hybrid approach is compared with other four techniques such as Threshold segmentation, $\mathrm{K}$ means clustering, Watershed segmentation, and Level set-based segmentation methods. Statistical and Visual analysis is performed. It is found that this novel hybrid approach has better specificity, accuracy, and precision among all four techniques. Further, it is able to detect tumors more accurately. This research could help clinicians in surgical planning, treatment planning and accurately segmenting the tumor part with the most accurate method.

\section{IMPLEMENTATION}

Step 1: Pre-processing of the input image by removing the Skull part and noise

1. The image is taken as input and the area taken by each component is found out. Label 0 is ignored since this is the background.

2. Get the label of the largest component by area

3. Get pixels that correspond to the brain

4. In a copy of the original image, clear those pixels that don't correspond to the brain to obtain the final images.

5. Then the image is converted into grayscale and is blurred using the median filter of Kernel size 5 to remove noise.

Step 2: Watershed segmentation is applied to the Pre-processed image of step 1 
1. Remove noise

2. Find the sure foreground area

3. Find the unknown region

4. Add 1 to all labels so that sure background is not 0

5. The region of the unknown is marked with zero

6. Finally, the watershed segmented image is plotted.

Step 3: Level set segmentation is applied to the watershed segmented image of step 2

1. Chan_vese method is used for detecting active contours

2. Finally, the Level set segmented image is plotted.

Step 4: K-means clustering is applied finally to the level set segmented image of step 3

1. First, the image is converted into RGB color space.

2. The RGB value of each pixel is vectorized to 3 columns

3. K-means clustering is performed using the criteria and k-means functions of OpenCV.

4. $\mathrm{K}$ value is chosen as 3 .

5. The Centers of the Cluster is obtained.

6. The final processed image after applying K-means is plotted.

Step 5: System accuracy, sensitivity, specificity, and precision are evaluated based on the following attributes.

- TP (True Positive): Existing tumor and detected correctly.

- $\quad$ TN (True Negative): Non-existing tumor and not detected.

- $\quad$ FP (False Positive): Non-existing tumor and detected.

- $\quad$ FN (False Negative): Existing tumor and not detected.

Step 6: Finally, the Statistical and Visual analysis is performed.

\section{RESULTS AND DISCUSSIONS}

A computer system with an Intel Core i5-7200U CPU @ 2.50GHz $2.71 \mathrm{GHz}$ processor is used for software implementation and the performance test. All of the segmentation techniques were simulated using PyCharm Community edition 2017.3.7 with Python as the programming language.

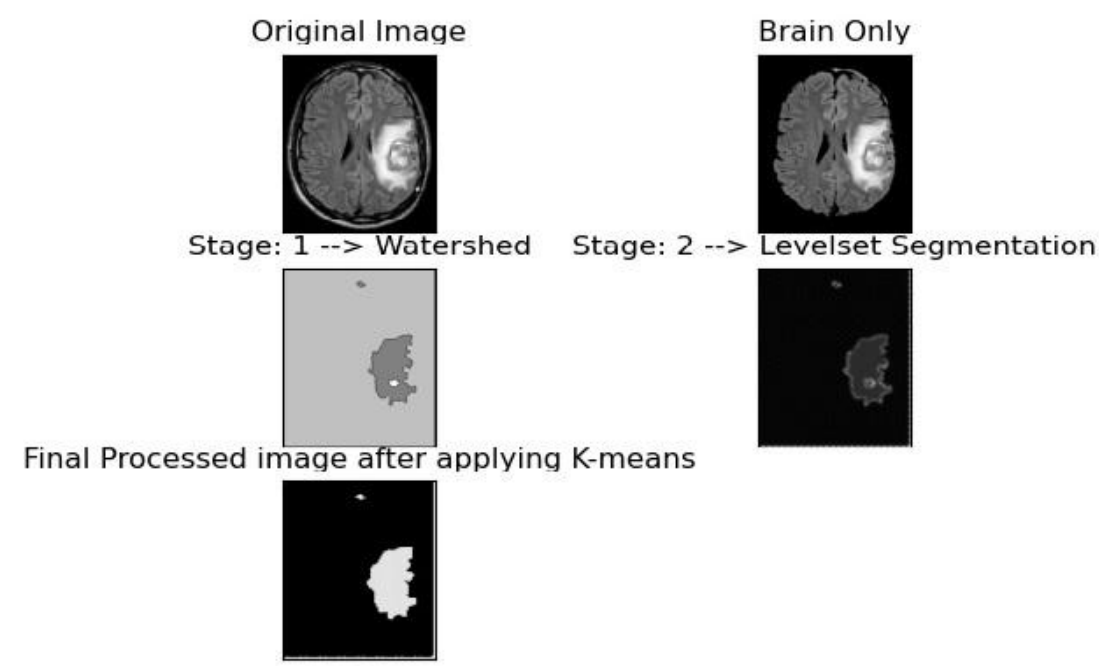

FIGURE 1: Final Processed image of Hybrid Approach.

Figure 1. is the Final Processed image of the Hybrid Approach. The dataset of MRI images of the Brain is taken from Kaggle [18]. In this work, a novel hybrid approach is implemented by combining watershed 
segmentation, level set segmentation and $\mathrm{k}$ means clustering. In this study, 80 tumor images and 20 non-tumor images are considered for implementation i.e., a total of 100 images in the dataset are tested.

Performance of hybrid segmentation approach is compared with four techniques such as Threshold segmentation, K means clustering, Watershed segmentation, Level set-based segmentation methods by calculating various parameters such as TP (True Positive) i.e., Existing tumor and detected correctly, TN (True Negative) i.e., Non-existing tumor and not detected, FP (False Positive) i.e., Non-existing tumor and detected and FN (False Negative) i.e. Existing tumor and not detected. Specificity, sensitivity, precision, and accuracy are calculated from the above parameters.

Sensitivity is the measure of successful determination of the person having a tumor.

$$
\text { Sensitivity }=\frac{T P}{T P+F N}
$$

Specificity is the measure of successful determination of the person not having a tumor.

$$
\text { Specificity }=\frac{T N}{T N+F P}
$$

Accuracy is the measure of successful classification.

$$
\text { Accuracy }=\frac{T P+T N}{T P+F P+T N+F N}
$$

\begin{tabular}{|c|c|c|c|c|c|c|c|c|}
\hline & $P^{T}$ & $\mathbf{N}^{\mathbf{T}}$ & $\mathbf{P}^{\mathbf{F}}$ & $\mathbf{N}^{\mathbf{F}}$ & ty $^{\text {Sensitivi }}$ & $\begin{array}{l}\text { Specific } \\
\text { ity }\end{array}$ & $\begin{array}{l}\text { Accur } \\
\text { acy }\end{array}$ & $\begin{array}{l}\text { Precis } \\
\text { ion }\end{array}$ \\
\hline $\begin{array}{r}\text { Threshold } \\
\text { Segmentation }\end{array}$ & 71 & $9^{1}$ & 1 & 9 & $88.75 \%$ & $95 \%$ & $90 \%$ & $\%$ \\
\hline K-means clustering & 65 & $9^{1}$ & 1 & $5^{1}$ & $81.25 \%$ & $95 \%$ & $84 \%$ & $\%$ \\
\hline $\begin{array}{c}\text { Watershed } \\
\text { Segmentation }\end{array}$ & 74 & $9^{1}$ & 1 & 6 & $92.5 \%$ & $95 \%$ & $93 \%$ & $\%$ \\
\hline Level Set Segmentation & 70 & $4^{1}$ & 6 & $0^{1}$ & $87.5 \%$ & $70 \%$ & $84 \%$ & $\%^{92.10}$ \\
\hline Novel Hybrid approach & 74 & $0^{2}$ & 0 & 6 & $92.5 \%$ & $100 \%$ & $94 \%$ & $100 \%$ \\
\hline
\end{tabular}

Precision is the measure of how many of those who are labeled as tumors are tumor.

$$
\text { Precision }=\frac{T P}{T P+F P}
$$

TABLE 1: The comparison of proposed hybrid approach for a total of 100 images.

Table 1 shows the comparison of the proposed hybrid approach for a total of 100 images. 80 images were tumor images and 20 were non-tumor images. Performance of hybrid segmentation approach is compared with four techniques such as Threshold segmentation, K means clustering, Watershed segmentation, Level set-based segmentation by calculating various parameters such as TP (True Positive), TN (True Negative), FP (False Positive), and FN (False Negative). Further specificity, sensitivity, precision, and accuracy are compared.

\section{CONCLUSION}

A novel hybrid approach is implemented by combing watershed segmentation, level set segmentation and $\mathrm{k}$ means clustering. First, the image is preprocessed by removing the skull. Watershed segmentation is applied to this preprocessed image. Level set segmentation is applied to the previous step. Finally, $\mathrm{k}$ means clustering is applied as the final step to detect tumor parts accurately. This Hybrid approach is compared with other four techniques such as Threshold segmentation, K means clustering, Watershed segmentation, and Level set-based segmentation methods. Statistical and Visual analysis is performed. It is found that this novel hybrid approach has better specificity, accuracy, and precision among all four techniques. Further, it is able to detect tumors more 
accurately. This research could help clinicians in surgical planning, treatment planning and accurately segmenting the tumor part with the most accurate method

\section{References}

R.A. Gonzalez, R.E. Woods, “Digital Image Processing”. Second Edition. Prentice Hall 2002

R. N. Strickland, "Image-Processing Techniques for Tumor Detection”, University of Arizona Tucson, Arizona, 2002

Natarajan P, Krishnan.N, Natasha S, Shraiya N, Bhuvanesh P, “Tumor Detection using threshold operation in MRI Brain Images", International Conference on Computational Intelligence and Computing Research, IEEE, 2012.

Manoj K and Sourabh Y, "Brain Tumor Detection and Segmentation Using Histogram Thresholding", International Journal of Engineering and Advanced Technology (IJEAT) ISSN: 2249 - 8958, Volume-1, Issue-4, April 2012.

M. Usman A, Anam. U. "Computer Aided system for Brain Tumor Detection and Segmentation" Computer Networks and information Technology (ICCNIT), IEEE, 2011.

G. M. N. R. Gajanayake, R. D. Yapa and B. Hewawithana, "Comparison of Standard Image Segmentation Methods for Segmentation of Brain Tumors from 2D MR Images”, 4th International Conference on Industrial and Information Systems, ICIIS, University of Peradeniya, Sri Lanka, pp. 301- 305., IEEE, 2009.

B. N. Saha, N. Ray, R. Greiner, A. Murtha, H. Zhang, "Quick detection of brain tumors and edemas: A bounding box method using symmetry", Computerized Medical Imaging and Graphics 36 (2012) 95- 107, Elsevier, 2011.

M. C. Clark, L. O. Hall, D. B. Goldgof, R. Velthuizen, F. Reed Murtagh, and M. S. Silbiger, “Automatic Tumor Segmentation Using Knowledge-Based Techniques", IEEE transactions on medical imaging, vol. 17, no. 2, April 1998.

C.L. Biji, D. Selvathi, and A. Panicker, "Tumor Detection in Brain Magnetic Resonance Images Using Modified Thresholding Techniques", A. Abraham et al. (Eds.): ACC 2011, Part IV, CCIS 193, pp. 300-308, 2011, Springer-Verlag Berlin Heidelberg, 2011.

J. Fan, G. Zeng, M. Body, M. Hacid, "Seeded region growing: an extensive and comparative study", Pattern Recognition Letters 26 1139-1156, Elsevier, 2005.

Yu-len huang and Dar-ren chen," Watershed segmentation for breast tumor in 2-d sonography", Ultrasound in Med. \& Biol., Vol. 30, No. 5, pp. 625-632, Elsevier, 2004.

N. Gordillo, E. Montseny, P. Sobrevilla, "State of the art survey on MRI brain tumor segmentation", journal of magnetic resonance imaging, Elsevier, 2013.

M. Letteboer, W. Niessen, P. Willems, E. B. Dam, and M. Viergever, "Interactive multi-scale watershed segmentation of tumors in mr brain images", in Proc. of the IMIVA Workshop of MICCAI, Citeseer, 2001

E. Dam, M. Loog, and M. Letteboer, "Integrating automatic and interactive brain tumor segmentation", in Pattern Recognition, 2004. ICPR 2004. Proceedings of the 17th International Conference on, IEEE, 2004, vol. 3, pp. 790- 793.

M. Masroor Ahmed \& Dzulkifli Bin Mohammad "Segmentation of Brain MR Images for Tumour Extraction by Combining Kmeans Clustering and Perona-Malik Anisotropic Diffusion Model”, oct 2007

S. Osher and J. A. Sethian, "Fronts propagating with curvature dependent speed: algorithms based on hamiltonjacobi formulations", Journal of Computational Physics, 79:12-49, 1988.

Tejas P and S. K. Padma, "Statistical Analysis of Segmentation Techniques for Brain Tumor Detection", International Journal of Latest Trends in Engineering and Technology, Vol. 17 ISSUE 3, pp. 36-40, Sep. 2020.

Navoneel Chakrabarty, "Brain MRI Images for Brain Tumor Detection”, USENET: https://www.kaggle.com/navoneel/brain-mri-images-for-brain-tumor-detection, 2018 